\title{
Glycopolymer Induction of Mouse Sperm Acrosomal Exocytosis Shows Highly Cooperative Self-Antagonism
}

\author{
Maria T. Rodolis, ${ }^{\mathrm{a}}$ He Huang, ${ }^{\mathrm{a}}$ and Nicole S Sampson ${ }^{\mathrm{a}, *}$
}

Short Title: Glycopolymer Acrosomal Exocytosis Assay

${ }^{a}$ Department of Chemistry, Stony Brook University, Stony Brook, NY, USA 11794-3400.

*Corresponding author: nicole.sampson@ stonybrook.edu (NSS) 


\section{ABSTRACT}

Identifying inducers of sperm acrosomal exocytosis (AE) to understand sperm functionality is important for both mechanistic and clinical studies in mammalian fertilization. Epifluorescence microscopy methods, while reproducible, are laborious and incompatible for high throughput screening. Flow cytometry methods are ideal for quantitative measurements on large numbers of samples, yet typically rely on the use of lectins that can interfere with physiologic AE-inducers. Here, we present an optimized triple stain flow cytometric method that is suitable for highthroughput screening of AE activation by glycopolymers. SYTO-17 and propidium iodide (PI) were used to differentiate cells based on their membrane integrity or viability, and membrane impermeable soybean trypsin inhibitor (SBTI) was used to monitor acrosome exocytosis. The SBTI/PI/SYTO-17 combination provides a positive screen for viability and AE of live sperm cells with minimal noise or false positives. A scattering gate enables the use of samples that may be contaminated with non-cellular aggregates, e.g., cryopreservation agents. This assay format enabled detailed analysis of glycopolymer dose response curves. We found that fucose polymer has a narrow effective dose range $\left(\mathrm{EC}_{50}=1.6 \mu \mathrm{M} ; \mathrm{IC}_{50}=13.5 \mu \mathrm{M}\right)$; whereas mannose polymer and $\beta$-N-acetylglucosamine polymer have broader effective dose ranges $\left(\mathrm{EC}_{50}=1.2 \mu \mathrm{M}\right.$ and 3.4 $\mu \mathrm{M}$, respectively). These results highlight the importance of testing inducers over a large concentration range in small increments for accurate comparison.

Keywords: Polymer, multivalent sugar, activation, sperm, flow cytometry 


\section{INTRODUCTION}

Sperm acrosomal exocytosis $\left(\mathrm{AE}^{1}\right)$ is a vital event for fertilization to occur [1]. Various physiological and nonphysiological agonists have been shown to activate mammalian AE including zona pellucida (ZP) and cumulus terminal monosaccharides [2-7]. Multiple spermcarbohydrate binding events must occur simultaneously to stimulate AE and identification of the associated receptors is complex [8-11]. Thus, synthetic biomaterials to assess sperm AE induction reproducibly are required to clearly identify receptors responsible, to develop simple semen analysis assays, and to identify new targets for contraceptives [11]. Several groups have used neoglycoconjugates to explore the involvement of individual types of saccharides in AE [12-14]. Previously, we found that a multivalent display of mannose, GlcNAc or fucose residues linked to a norbornene-derived polymer backbone triggers $\mathrm{AE}$ in a concentration dependent manner, and the signaling induced by these polymers converges onto the same intracellular signaling pathways [14]. The chemotypes suggest that single ligand type-receptor interactions are equivalent, but redundant, and further study was warranted. In order to further develop these glycopolymers as probes of $\mathrm{AE}$, we developed the flow cytometry method described in this work as a robust and reliable assay that can be used to analyze larger numbers of samples that utilize a neoglycopolymer as the AE-inducer.

Our original studies, like many others, were performed utilizing Peanut agglutinin (PNA), lectin staining and a combined epifluorescence and DIC microscopy assay. Although highly reproducible, assessment of AE by combined fluorescence and DIC microscopy is laborious and incompatible with high-throughput analysis. In addition, it is difficult to differentiate healthy

\footnotetext{
${ }^{1}$ Abbreviations: AE: acrosomal exocytosis; ZP, zona pellucida; GlcNAc: N-acetylglucosamine; DIC, differential interference contrast; Gal, galactose; PNA: peanut agglutinin; IAM, inner acrosomal membrane: SBTI: soybean trypsin inhibitor; PI, propidium iodide; Glu, glucose.
} 
acrosome exocytosed sperm from damaged sperm after fixing and lectin staining. In order to analyze the activities of a large set of related glycopolymers, we set out to explore an alternative method to assess simultaneously AE and sperm viability.

Triple-stain flow cytometry methods to evaluate sperm viability and acrosome integrity have been developed $[15,16]$. Using a combination of membrane permeable and impermeable DNA binding dyes such as SYTO-17 and propidium iodide (PI), respectively, with an acrosomespecific lectin provides a method of distinguishing viable from damaged cells by flow cytometry, eliminates non-cellular aggregates that can be present in samples, and identifies sperm that have undergone AE [17-19]. PNA, often used in AE assessment, is a membrane impermeable lectin that binds to soluble acrosomal molecules that contain a Gal- $\beta(1-3)$-GalNAc sugar sequence $[18,20]$. Membrane impermeable PNA can only bind to its ligand when cells are at the early stage of acrosome exocytosis [21]. As exocytosis progresses, ligand bound-PNA diffuses out of the cell and the fluorescent signal is lost. The kinetics of AE have been previously shown to vary across individuals [22], therefore utilizing the time-sensitive lectin PNA on live cells is unsuitable for high-throughput analysis. Membrane fixation and permeabilization is necessary in order for PNA to bind to acrosomal ligands of intact cells [7, 21, 23], and thus, viability is not evaluated. The combination of Pisum sativum agglutinin (PSL) and PI was used to assess AE in live human sperm.[24] In contrast to use of PSL with fixed sperm, PSL diffuses into the acrosome after exocytosis begins and pores open, and upon binding, stabilizes the acrosome matrix. Thus PSL provides a positive fluorescence signal for $\mathrm{AE}$ in live sperm that is persistent for greater than ten minutes, thereby simplifying detection. However, PSL works through its high affinity interaction with mannose and glucose. The use of lectins in general is problematic with saccharide-based AE inducers in a live monitoring assay. Because the recognition elements for lectins are sugars, AE- 
inducers that utilize glycoproteins from ZP or glycopolymers can interfere with lectin recognition of sperm AE. Thus, the currently established staining systems were insufficient for our purposes.

A number of proteases have been identified in the acrosome of mouse sperm including acrosin, an endoprotease with trypsin-like specificity located on the inner acrosomal membrane (IAM) $[25,26]$. Acrosin is derived from the enzymatically inactive zymogen, proacrosin, which is converted to the active form as a result of AE [27]. Soybean trypsin inhibitor (SBTI) inhibits the catalytic activity of serine proteases, including acrosin, by directly binding to the protease [28]. Localization of Alexa Fluor ${ }^{\circledR} 488$ SBTI revealed that binding increases rapidly as the IAM is exposed during AE [29]. This increase is followed by a slow decline in fluorescence representing the release of non-membrane associated acrosin into the surrounding medium. Ultimately the fluorescence signal from remaining IAM-bound acrosin stabilizes [30, 31]. The stable SBTI fluorescence at a raised plateau provides a larger window of time for measuring AE reproducibly. Therefore, we set out to develop and optimize a triple stain flow cytometric method using SBTI, SYTO-17 and PI that is suitable for high-throughput screening and that would produce results comparable to our previously published microscopy data. This method was used to assess the AE activity of glycopolymers with varying structures and lays the groundwork for $\mathrm{AE}$ activation assays with clinical samples. 


\section{MATERIALS AND METHODS}

All experiments performed on mice were approved by the Stony Brook University IACUC (Protocol 0616) and were conducted in accordance with the National Institute of Health and the United States Department of Agriculture guidelines. DMSO and A23187 calcium ionophore were purchased from Sigma-Aldrich. A23187 stock solution $(1 \mathrm{mg} / \mathrm{ml})$ was prepared by dilution with DMSO. Propidium iodide was purchased from Fisher Scientific. Alexa Fluor ${ }^{\circledR} 488$ soybean trypsin inhibitor (SBTI) and SYTO-17 were purchased from Life Technologies. All chemicals for assay buffers were purchased from Sigma-Aldrich, Fisher Scientific and VWR.

Fluorescent Stains. Propidium iodide (PI) was dissolved in water (2.4 mM) and stored at $20{ }^{\circ} \mathrm{C}$. Alexa Fluor ${ }^{\circledR} 488$ soybean trypsin inhibitor (SBTI) was dissolved in water $(1 \mathrm{mg} / \mathrm{ml})$ and stored at $-20{ }^{\circ} \mathrm{C}$ in $40 \mu \mathrm{l}$ aliquots. SYTO-17 was diluted in DMSO $(1 \mathrm{mM})$ and stored at $-20{ }^{\circ} \mathrm{C}$.

Glycopolymer solutions. Deprotected polymers were synthesized and characterized as reported [14]. Stock solutions of poly $(\mathrm{Glu})_{100}$, poly $(\mathrm{Gal})_{100}$, poly(GalNAc) ${ }_{100}$, poly(Man $)_{100}$, poly(Fuc $)_{100}$ and poly $(\mathrm{GlcNAc})_{100}$ were stored in DDI water at $-20{ }^{\circ} \mathrm{C}$ at a polymer concentration of $100 \mu \mathrm{M}$.

Sperm Treatment. Sperm were isolated by force from the cauda epididymis of two 9- to 12-week-old CD-1 male breeders (Charles River) in a phenol red-free M16 medium (2.57 mM $\left.\mathrm{CaCl}_{2}\right)(6 \mathrm{~mL})$ supplemented with $0.3 \% \mathrm{BSA}(\mathrm{w} / \mathrm{v})$. The sperm suspension was then gently pipetted into a polypropylene culture tube $(12 \mathrm{~mm} \times 75 \mathrm{~mm})$ and incubated for $10 \mathrm{~min}$ at $25{ }^{\circ} \mathrm{C}$. Then the concentration of sperm concentration was assessed by haemocytometer and motility was examined by phase-contrast microscopy (20X). Aliquots $(125 \mu 1)$ containing about $2.5 \times 10^{6}$ sperm were subsequently diluted to a final volume of $250 \mu 1\left(1.25 \times 10^{7} \mathrm{sperm} / \mathrm{ml}\right)$. The sperm suspension was incubated for another $15 \mathrm{~min}$ to get additional capacitation at $25{ }^{\circ} \mathrm{C}$. A $250 \mu \mathrm{l}$ solution 
containing SYTO $17(2.5 \mu \mathrm{l})$, SBTI $(1 \mu \mathrm{l})$ and either A23187 (5 $\mu \mathrm{M}$, positive control), glycopolymer $(0.5 \mu \mathrm{M}-30 \mu \mathrm{M}$, sample), or Dulbecco's Phosphate-Buffered Saline (PBS, negative control) was added to the $250 \mu \mathrm{l}$ sperm suspension to a final volume of $500 \mu \mathrm{l}$. The mixture was kept at $0{ }^{\circ} \mathrm{C}$ in the dark for $15 \mathrm{~min}$ and then $20 \mathrm{~min}$ in the dark at $\mathrm{rt}$. Samples were centrifuged for $5 \mathrm{~min}$ at $500 \mathrm{~g}$, and the resulting pellet was re-dissolved with $500 \mu \mathrm{l}$ of a PBS solution containing PI $(24 \mu \mathrm{M})$, SBTI $(2 \mu \mathrm{g} / \mathrm{ml})$ and SYTO-17 $(5 \mu \mathrm{M})$. Samples were resuspended at $1 \mathrm{~min}$ intervals and allowed to incubate for $20 \mathrm{~min}$ at $25{ }^{\circ} \mathrm{C}$ before flow cytometric analysis.

Flow Cytometry. Analyses were performed on a Becton Dickinson LSR Fortessa system in combination with the BD-FACSDiva ${ }^{\mathrm{TM}}$ Software Version 8. SYTO-17 stained all cellular events and was excited at $640 \mathrm{~nm}$ and detected at 670/30 $\mathrm{nm}$. PI stained all nonviable cells and was excited at $561 \mathrm{~nm}$ and detected at 582/15 nm. Alexa Fluor ${ }^{\circledR} 488$ SBTI stained all cells that have undergone acrosome exocytosis and was excited at $488 \mathrm{~nm}$ and detected at 530/30 $\mathrm{nm}$. These three stains have minimal emission overlap. Utilizing the forward and sideways scatter, small debris was gated out of analyses. As previously reported, additional gating was necessary to remove noncellular events which displayed similar scatter characteristics to sperm [16]. Events which exhibited weak SYTO-17 and PI staining (weak DNA content) were gated out before acrosome integrity and viability could be determined. Viability and acrosome integrity was measured at a flow rate of 600 cells/sec for $1 \mathrm{~min}$. On average, $20 \%$ of sperm were PI negative (alive). $9 \%$ of live sperm cells undergo spontaneous acrosome exocytosis based on the negative control. In the presence of $5 \mu \mathrm{M}$ A23187, approximately $21 \%$ of live cells undergo acrosome exocytosis. AE induced by A23187 was concentration dependent. However, at high concentrations, a large 
amount of cell death was observed. In addition, excess cellular debris complicates gating at high concentrations. Therefore, $5 \mu \mathrm{M}$ A23187 was selected for use as a positive control.

Statistical Analysis. The acrosome integrity of live sperm was normalized to PBS (negative control) and $5 \mu \mathrm{M} \mathrm{A23187} \mathrm{(positive} \mathrm{control).} \mathrm{Only} \mathrm{live} \mathrm{sperm} \mathrm{were} \mathrm{included} \mathrm{in} \mathrm{the} \mathrm{analysis} \mathrm{and}$ each experiment was conducted in biological triplicate. Normalized AE\% was calculated using $[\mathrm{AE} \%$ of glycopolymers $-\mathrm{AE} \%$ of negative control)]/[AE\% positive control - $\mathrm{AE} \%$ negative control]. Data represent mean \pm SEM of at least three independent experiments when compared to the negative control. $\mathrm{EC}_{50}$ was obtained by fitting equation (1) and $\mathrm{IC}_{50}$ was obtained by fitting equation (2) using GraphPad or Kaleidagraph to the data:

$$
\begin{aligned}
& \mathrm{AE} \%=\mathrm{AE}_{\min }+\left(\mathrm{AE}_{\max }-\mathrm{AE}_{\min }\right) /\left\{1+10^{\wedge\left((\log \mathrm{EC} 50)-\log ([\text { polymer }])^{*}\right)}\right\} \\
& \mathrm{AE} \%=\mathrm{AE}_{\min }+\left(\mathrm{AE}_{\max }-\mathrm{AE}_{\min }\right) /\left\{1+10^{\wedge((\log \mathrm{C} 50)+\log ([\text { polymer }]) * \mathrm{~s})}\right\}
\end{aligned}
$$

Where $\mathrm{AE}_{\max }$ is the maximum percentage of $\mathrm{AE}$ observed, $\mathrm{AE}_{\min }$ is the lowest percentage of $\mathrm{AE}$ observed, $\mathrm{EC}_{50}$ is the concentration at which $50 \% \mathrm{AE}$ is observed, and $\mathrm{s}$ is the slope of the response. For the poly(Fuc) $)_{100}$ data, the $\mathrm{EC}_{50}$ and $\mathrm{IC}_{50}$ response curves were fit independently to the corresponding subset of data that encompassed the activation or inhibition arm of the curve. The average $\mathrm{AE}_{\max }$ and $\mathrm{AE}_{\min }$ from these two curve fits was then used to refit the data to equations (3) and (4) to determine $\mathrm{EC}_{50}$ and $\mathrm{IC}_{50}$, respectively. Data represent mean $\pm \mathrm{SEM}$ of between two and four independent experiments.

$$
\begin{aligned}
& \mathrm{AE} \%=5.3+(14.2) /\left\{1+10^{\wedge((\operatorname{logEC} 50)-\log ([\mathrm{polymer}]) * \mathrm{~s})}\right\} \\
& \mathrm{AE} \%=5.3+(14.2) /\left\{1+10^{\wedge((\log I \mathrm{C} 50)+\log ([\mathrm{polymer}]) * \mathrm{~s})}\right\}
\end{aligned}
$$




\section{RESULTS}

Gating analysis of triply stained sperm. After AE induction, sperm were labeled with SYTO-17, PI and Alexa Fluor 488 SBTI and analyzed by flow cytometry for viability and acrosome integrity. Gating was conducted in a similar manner to previously published methods [16], in which a two-dimensional dot plot of the side versus the forward scatter was used to remove small debris from the analysis, and a subsequent two-dimensional dot plot of PI vs SYTO17 was used to remove non-cellular events from the analysis (Fig. 1, A and B, respectively). The number of live-acrosome-reacted sperm was determined from the two dimensional dot plot of SBTI versus PI and the resulting two dimensional histogram for SBTI staining of PI negative (live) cells (Fig. 1, C, D, and E respectively).

\section{[Fig. 1]}

Live acrosome-exocytosed cells exhibited high SBTI fluorescence emission resulting in the appearance of a new distinct peak on the SBTI histogram (Fig. 1, C and E). In some instances, this peak was flat and broad. However, acrosome-intact and acrosome-exocytosed sperm were distinguished by comparison with the control samples.

Induction of AE by glycopolymers. AE induction by six glycopolymers was measured at 5 $\mu \mathrm{M}, 10 \mu \mathrm{M}$ and $20 \mu \mathrm{M}$ by flow cytometry. The levels of $\mathrm{AE}$ induced were equivalent to those measured by fluorescence microscopy (Fig. 2). The high AE inducers poly(Man) ${ }_{100}$, poly $(\text { GlcNAc })_{100}$ and poly(Fuc) $)_{100}$ show small differences in the maximal induction achieved. With the higher throughput of the flow cytometry assay, we undertook investigation of the activity of these AE-inducing glycopolymers across a larger range of concentrations in smaller steps (Fig. 3, Table 1). Polymers were not tested above $20 \mu \mathrm{M}$ as sperm viability was reduced upon addition of high concentrations of polymer. 


\section{[Fig. 2]}

\section{[Fig. 3]}

\section{[Table 1]}

\section{DISCUSSION}

Our requirements for a flow cytometry based analysis of acrosomal exocytosis were twofold. First, we required a gain of signal indicator of AE. Second, we required an AE probe that would not succumb to interference by a variety of glycopolymers. Previous methods have described the evaluation of acrosome integrity with SBTI and PI [32]. Because the SBTI ligand is a protein, we reasoned that glycopolymer AE inducers would not interfere with a live sperm labelling assay. We explored dual staining with SBTI and PI. However, in the absence of a DNA content gating protocol, i.e., SYTO-17/PI (Fig. 1B), a significant amount of sperm-sized debris or non-cellular events was included in the final analysis. In the absence of the SYTO-17 gating, up to $70 \%$ of events tagged as "live sperm" were counted as acrosome exocytosed in the positive control (5 $\mu \mathrm{M}$ A23187). In contrast, analysis of equivalent samples by microscopy identified approximately $25 \%$ of fixed sperm as having undergone AE. With the SYTO-17 gating step, noncellular events were eliminated from the analysis. This method identified a similar fraction of cellular events to that observed using PNA/SYBR-14/PI [17].

We selected an SBTI staining time of 20 min in order to balance the stability of the signal and to minimize the level of spontaneous $\mathrm{AE}$ observed in negative controls. Live sperm percentage was the same at $25{ }^{\circ} \mathrm{C}$ in ambient air and at $37{ }^{\circ} \mathrm{C}$ with $5 \% \mathrm{CO}_{2}$ consistent with previous reports.[33] We selected $25^{\circ} \mathrm{C} / \mathrm{ambient}$ air as the incubation temperature to minimize spontaneous $\mathrm{AE}$ and to facilitate handling of samples. 
$\mathrm{Wu}$ and Sampson [14] previously demonstrated using inverse fluorescence and DIC microscopy that homoglycopolymers displaying 100 copies of mannose, GlcNAc or fucose along a norbornyl backbone induce mouse sperm AE in a concentration dependent manner. The same polymer batches used by $\mathrm{Wu}$ and Sampson [14] were retested with the flow cytometry assay described here. The normalized AE percentages measured for these polymers are equivalent to our previously published fluorescence microscopy data.

Analysis by flow cytometry allowed us to measure the activity of these polymers with smaller concentration increments across a wide range of concentrations. The concentration studies provided full dose response $\mathrm{AE}$ curves that revealed poly(NBFuc) ${ }_{100}$ is the most effective $\mathrm{AE}$ inducer (Figure 3A). The differences in the shape of these three dose-response curves highlight the importance of measuring concentration dependence for polyvalent inducers in small step sizes in order to avoid missing the effective dose range of inducers. At concentrations above $10 \mu \mathrm{M}$ poly(NBFuc) ${ }_{100}$, highly cooperative self-antagonism is observed. This inhibition is characteristic of a receptor that requires multivalent engagement of receptor [34-37]. The inhibition arm may result from competition of the multivalent interaction with monovalent binding $\left(\mathrm{K}_{1} \mathrm{vs}_{\mathrm{K}}\right.$, Figure 3B). In addition, steric occlusion may prevent engagement of every ligand on the polymer chain. However, the inhibition response is steeper than expected for either or both cases [37]. We hypothesize that the high cooperativity of inhibition results from the formation of a larger number of receptor-polymer multivalent complexes that $\underline{d o} \underline{n o t}$ activate signaling $\left(\mathrm{K}_{2}{ }^{\prime}\right)$ than of complexes that $\underline{d o}$ activate signaling $\left(\mathrm{K}_{2}\right)$ (Figure $\left.3 \mathrm{~B}\right)$. The consequence is that the effective dose range is quite narrow.

The $\mathrm{EC}_{50}$ for poly(NBMan) $)_{100}$ is identical to that of poly(NBFuc) ${ }_{100}$ and no inhibition is observed. Despite a broader effective dose range, the maximal AE induction observed is lower 
than for fucose, indicating that not all complexes formed initiate AE signaling. poly(NBGlcNAc) ${ }_{10}$ was less effective than fucose both in $\mathrm{EC}_{50}$ and maximal response, and poly(NBGlcNAc) ${ }_{100}$ shows the beginning of antagonism at the highest concentrations measurable.

The differences in the shape of these three dose-response curves for these polymers highlight the importance of measuring concentration dependence for polyvalent inducers in small step sizes in order to avoid missing the effective dose range of inducers/inhibitors. The dose responses are consistent with our previous conclusion that the three polymers activate $\mathrm{AE}$ through different receptors that converge into a single signaling pathway [14]. Further interpretation of the physiologic significance awaits identification of the receptors that are engaged and the structures of the receptor-polymer complexes [38].

The triple stain flow cytometric assay discussed in this study provides a quantitative method to inform on sperm viability and acrosome integrity in the presence of exocytosis inducers and/or inhibitors. The rapid throughput format opens the possibility of testing libraries of compounds to both investigate reasons for infertility and ways to block acrosome exocytosis. Ultimately, these types of probes will serve as platforms for infertility testing.

\section{Acknowledgment}

We thank Bart Gadella, Pablo Visconti, Toshiaki Hino, and the SBU Flow Cytometry Facility staff for their helpful discussion of the flow cytometry assay and Linghui Wu for providing polymer samples. Supported by funding from the NIH: GM097971 (N.S.S.) and NSF: HRD 1311318 (M.R.). 


\section{References}

[1] P.M. Wassarman, L. Jovine, E.S. Litscher, H. Qi, Z. Williams, Egg-sperm interactions at fertilization in mammals, Eur. J. Obstet. Gynaecol. Reprod. Biol., 115 Suppl (2004) S57-60.

[2] J.C. Kirkman-Brown, L. Emma, C.L.R. Barratt, S.J. Publicover, Zona pellucida and progesterone-induced $\mathrm{Ca} 2+$ signaling and acrosome reaction in human spermatozoa, J. Androl., 23 (2002) 306-315.

[3] B. Baibakov, L. Gauthier, P. Talbot, T.L. Rankin, J. Dean, Sperm binding to the zona pellucida is not sufficient to induce acrosome exocytosis, Development, 134 (2007) 933-943.

[4] D.J. Miller, X. Gong, B.D. Shur, Sperm require N-acetylglucosaminidase to penetrate through the egg zona pellucida, Development, 118 (1993) 1279-1289.

[5] S.K. Nagdas, Y. Araki, C.A. Chayko, M.C. Orgebin-Crist, D.R. Tulsiani, O-Linked trisaccharide and $\mathrm{N}$-linked poly- $\mathrm{N}$-acetyllactosaminyl glycans are present on mouse ZP2 and ZP3, Biol. Reprod., 51 (1994) 262-272.

[6] G.A. Cornwall, D.R. Tulsiani, M.C. Orgebin-Crist, Inhibition of the mouse sperm surface alpha-D-mannosidase inhibits sperm-egg binding in vitro, Biol. Reprod., 44 (1991) 913-921. [7] A. Dell, S. Chalabi, R.L. Easton, S.M. Haslam, M. Sutton-Smith, M.S. Patankar, F. Lattanzio, M. Panico, H.R. Morris, G.F. Clark, Murine and human zona pellucida 3 derived from mouse eggs express identical O-glycans, Proc. Natl. Acad. Sci. U. S. A., 100 (2003) 15631-15636.

[8] G.F. Clark, A role for carbohydrate recognition in mammalian sperm-egg binding, Biochem Biophys Res Commun, 450 (2014) 1195-1203.

[9] D.R. Tulsiani, Carbohydrates mediate sperm-ovum adhesion and triggering of the acrosome reaction, Asian J Androl, 2 (2000) 87-97. 
[10] P.M. Wassarman, Contribution of mouse egg zona pellucida glycoproteins to gamete recognition during fertilization, J. Cell. Physiol., 204 (2005) 388-391.

[11] P.C. Chiu, K.K. Lam, R.C. Wong, W.S. Yeung, The identity of zona pellucida receptor on spermatozoa: an unresolved issue in developmental biology, Seminars in cell \& developmental biology, 30 (2014) 86-95.

[12] C.R. Loeser, D.R. Tulsiani, The role of carbohydrates in the induction of the acrosome reaction in mouse spermatozoa, Biol. Reprod., 60 (1999) 94-101.

[13] W.F. Hanna, C.L. Kerr, J.H. Shaper, W.W. Wright, Lewis X-containing neoglycoproteins mimic the intrinsic ability of zona pellucida glycoprotein $\mathrm{ZP} 3$ to induce the acrosome reaction in capacitated mouse sperm, Biol. Reprod., 71 (2004) 778-789.

[14] L. Wu, N.S. Sampson, Fucose, mannose, and beta-N-acetylglucosamine glycopolymers initiate the mouse sperm acrosome reaction through convergent signaling pathways, ACS Chem. Biol., 9 (2014) 468-475.

[15] C.A. Thomas, D.L. Garner, M. Dejarnette, C.E. Marshall, T.E.T. Al, Fluorometric assessments of acrosomal integrity and viability in cryopreserved bovine spermatozoa, Biol. Reprod., 56 (1997) 991-998.

[16] S. Nagy, J. Jansen, E.K. Topper, B.M. Gadella, A triple-stain flow cytometric method to assess plasma- and acrosome-membrane integrity of cryopreserved bovine sperm immediately after thawing in presence of egg-yolk particles., Biol. Reprod., 68 (2003) 1828-1835.

[17] B.S. Edwards, T. Oprea, E.R. Prossnitz, L.A. Sklar, Flow cytometry for high-throughput, high-content screening., Curr. Opin. Chem. Biol., 8 (2004) 392-398. 
[18] S. Apostolski, S.A. Sadiq, A. Hays, M. Corbo, P. Chaliff, K. Stefansson, R.G. Lebaron, E. Ruoslahti, A.P. Hays, N. Latov, Identification of Gal ( $\beta-3)$ GalNAc bearing glycoproteins at the nodes of ranvier in peripheral nerve, J. Neurosicence, 38 (1994) 134-141.

[19] M.S. Hossain, A. Johannisson, M. Wallgren, S. Nagy, A.P. Siqueira, H. Rodriguez-Martinez, Flow cytometry for the assessment of animal sperm integrity and functionality: state of the art., Asian J. Androl., 13 (2011) 406-419.

[20] M.R.M. Hussain, M. Hassan, I. Afzal, A. Afzal, Role of Gal and GalNAc containing glycans in various physiological processes, Egypt. J. Med. Hum. Genetics, 13 (2012) 1-9. [21] P.F.N. Silva, B.M. Gadella, Detection of damage in mammalian sperm cells., Theriogenology, 65 (2006) 958-978.

[22] K. Takahashi, A. Wetzels, H. Goverde, B. Bastaans, H. Janssen, R. Rolland, The kinetics of the acrosome reaction of human spermatozoa and its correlation with in vitro fertilization, Fertil. Steril., 57 (1992) 889-894.

[23] S.S. Baker, R.A. Cardullo, C.D. Thaler, Sonication of Mouse Sperm Membranes Reveals Distinct Protein Domains, Biol. Reprod., 64 (2002) 57-64.

[24] F.C. Zoppino, N.D. Halon, M.A. Bustos, M.A. Pavarotti, L.S. Mayorga, Recording and sorting live human sperm undergoing acrosome reaction, Fertil. Steril., 97 (2012) 1309-1315. [25] M. Ferrer, H. Rodriguez, L. Zara, Y. Yu, W. Xu, R. Oko, MMP2 and acrosin are major proteinases associated with the inner acrosomal membrane and may cooperate in sperm penetration of the zona pellucida during fertilization., Cell Tissue Res., 349 (2012) 881-895. [26] H.T. Mao, W.X. Yang, Modes of acrosin functioning during fertilization, Gene, 526 (2013) 75-79. 
[27] K. Nayernia, I. Adham, R. Shamsedin, W. Engel, The role of acrosin in reproduction, J. Reprod. Infertil., 1 (2000) 38-43.

[28] A.I. Yudin, C.A. Vandevoort, M.-w. Li, J.W. Overstreet, PH-20 but not acrosin is involved in sperm penetration of the macaque zona pellucida, Mol. Reprod. Dev., 53 (1999) 350-362.

[29] T.L. Tollner, A.I. Yudin, G.N. Cherr, J.W. Overstreet, Soybean trypsin inhibitor as a probe for the acrosome reaction in motile cynomolgus macaque sperm, Zygote, 8 (2000) 127-137. [30] C.V. Harper, J.A. Cummerson, M.R. White, S.J. Publicover, P.M. Johnson, Dynamic resolution of acrosomal exocytosis in human sperm, J. Cell Sci., 121 (2008) 2130-2135. [31] K.-s. Kim, J.A. Foster, G.L. Gerton, Differential release of guinea pig sperm acrosomal components, Biol. Reprod., 64 (2001) 148-156.

[32] H. Tateno, D. Krapf, T. Hino, C. Sánchez-Cárdenas, A. Darszon, R. Yanagimachi, P.E. Visconti, Ca2+ ionophore A23187 can make mouse spermatozoa capable of fertilizing in vitro without activation of cAMP-dependent phosphorylation pathways., Proc. Natl. Acad. Sci. U. S. A., 110 (2013) 18543-18548.

[33] R. Matsuura, T. Takeuchi, A. Yoshida, Preparation and incubation conditions affect the DNA integrity of ejaculated human spermatozoa, Asian J. Androl., 12 (2010) 753-759.

[34] A. Whitty, C.W. Borysenko, Small molecule cytokine mimetics, Chem. Biol., 6 (1999) R107118.

[35] E.T. Mack, R. Perez-Castillejos, Z. Suo, G.M. Whitesides, Exact analysis of ligand-induced dimerization of monomeric receptors, Anal Chem, 80 (2008) 5550-5555.

[36] A. Fegan, B. White, J.C.T. Carlson, C.R. Wagner, Chemically Controlled Protein Assembly: Techniques and Applications, Chem. Reviews, 110 (2010) 3315-3336. 
[37] M.I. Monine, R.G. Posner, P.B. Savage, J.R. Faeder, W.S. Hlavacek, Modeling multivalent ligand-receptor interactions with steric constraints on configurations of cell-surface receptor aggregates, Biophys J, 98 (2010) 48-56.

[38] M. Atanasova, A. Whitty, Understanding cytokine and growth factor receptor activation mechanisms, Crit Rev Biochem Mol Biol, 47 (2012) 502-530. 
TABLE LEGEND

TABLE 1. Dose response constants for polymer inducers of AE. ${ }^{1}$

\section{FIGURE LEGENDS}

FIGURE 1. Gating procedures for AE analysis using scatter properties and staining. Immediately after centrifugation, the cell suspension was diluted in PBS and stained with SYTO-17, PI and Alexa Fluor 488 SBTI as described in Materials and Methods. Representative data in A) and B) are shown for sperm treatment with $10 \mu \mathrm{M}$ poly(Fuc) ${ }_{100}$. A) Gate 1: Two dimensional dot plot of side- versus forward scatter utilized to remove small debris from the analysis. B) Gate 2: Two dimensional dot plot of PI versus SYTO-17 utilized to remove non-cellular events from analysis. Depending on the level of debris, events with a SYTO-17 intensity over $10^{3}$ were typically gated for further analysis. C) D) and E) Analysis for acrosome integrity by two-dimensional dot plot of PI and SBTI staining of cellular events and the corresponding histogram of number of PI negative (live) cells versus SBTI fluorescence intensity. PI positive cells are shown in blue, have a disrupted membrane and are considered dead (acrosome intact and acrosome reacted); PI negative/SBTI negative cells are shown in purple and have an intact acrosome and plasma membrane and are considered live. PI negative/SBTI positive cells are shown in green and have an intact plasma membrane and have undergone acrosome exocytosis. The populations of sperm cells considered acrosomal exocytosed are represented by the higher SBTI fluorescence intensity on the histograms. Events shown in black or red represent noncellular events and/or debris. C) Upon treatment with $10 \mu \mathrm{M}$ poly(Fuc $)_{100}, 19.3 \%$ of PI negative (live) cells undergo AE. D) Upon treatment with PBS (negative control), 9.1\% of PI negative (live) cells undergo AE. E) Upon treatment with $5 \mu \mathrm{M}$ A23187 (positive control), 22.9\% of PI negative (live) cells undergo AE. 
FIGURE 2. Comparison of epifluorescence microscopy [14] and flow cytometry assays for the assessment of the activation of AE by glycopolymers. Average acrosome reaction percentages were normalized using [AE $\%$ of glycopolymers - AE $\%$ of negative control)]/[AE\% positive control - AE\% negative control]. The average AE\% for sperm treated with $5 \mu \mathrm{M} \mathrm{A} 23187$ (positive control) was $21 \%$. The average AE\% for sperm treated with PBS (negative control) was 9\%. Data represent mean \pm SEM of at least three independent experiments. A) poly $\left.(\mathrm{Glc})_{100}, \mathrm{~B}\right)$, $\left.\left.\left.\left.\operatorname{poly}(\mathrm{Gal})_{100}, \mathrm{C}\right) \operatorname{poly}(\mathrm{GalNAc})_{100}, \mathrm{D}\right) \operatorname{poly}(\mathrm{Man})_{100}, \mathrm{E}\right), \operatorname{poly}(\mathrm{Fuc})_{100}, \mathrm{~F}\right) \operatorname{poly}(\mathrm{GlcNAc})_{100}$.

FIGURE 3. A) Dose response curves for active homoglycopolymers poly(Fuc) ${ }_{100}$, poly(GlcNAc) ${ }_{100}$, and poly(Man $)_{100 .}$. AE values determined by flow cytometry are not normalized. Data represent mean \pm SEM of at least three independent biological replicates. In the case of poly $(\mathrm{Fuc})_{100}$ the $\mathrm{EC}_{50}$ and $\mathrm{IC}_{50}$ curves were fit separately. B) Model for polyvalent binding. $\mathrm{K}_{1}$ : dissociation constant for monovalent binding; $\mathrm{K}_{2}$ : dissociation constant for bivalent AE-induction; $\mathrm{K}_{2}$ ': dissociation constant for bivalent AE-antagonism. Higher orders of multivalency are possible. 


\section{Table 1}

\begin{tabular}{lccc} 
Polymer & $\mathrm{EC}_{50}(\mu \mathrm{M})$ & $\mathrm{IC}_{50}(\mu \mathrm{M})$ & $\mathrm{S}$ \\
\hline poly(Fuc) & & & \\
& $1.6 \pm 1.1$ & $13.5 \pm 1.0$ & $1.5 \pm 0.2,-9.5 \pm 3.0$ \\
poly(GlcNAc) $)_{100}$ & $3.4 \pm 2.2$ & $\mathrm{ND}^{2}$ & $0.9 \pm 0.6$ \\
poly(Man $)_{100}$ & $1.2 \pm 1.7$ & $\mathrm{ND}$ & $1.3 \pm 0.8$
\end{tabular}

${ }^{1}$ Data are fit as described in Materials and Methods. Errors are the standard error of the fit. ${ }^{2}$ ND: not determined. 


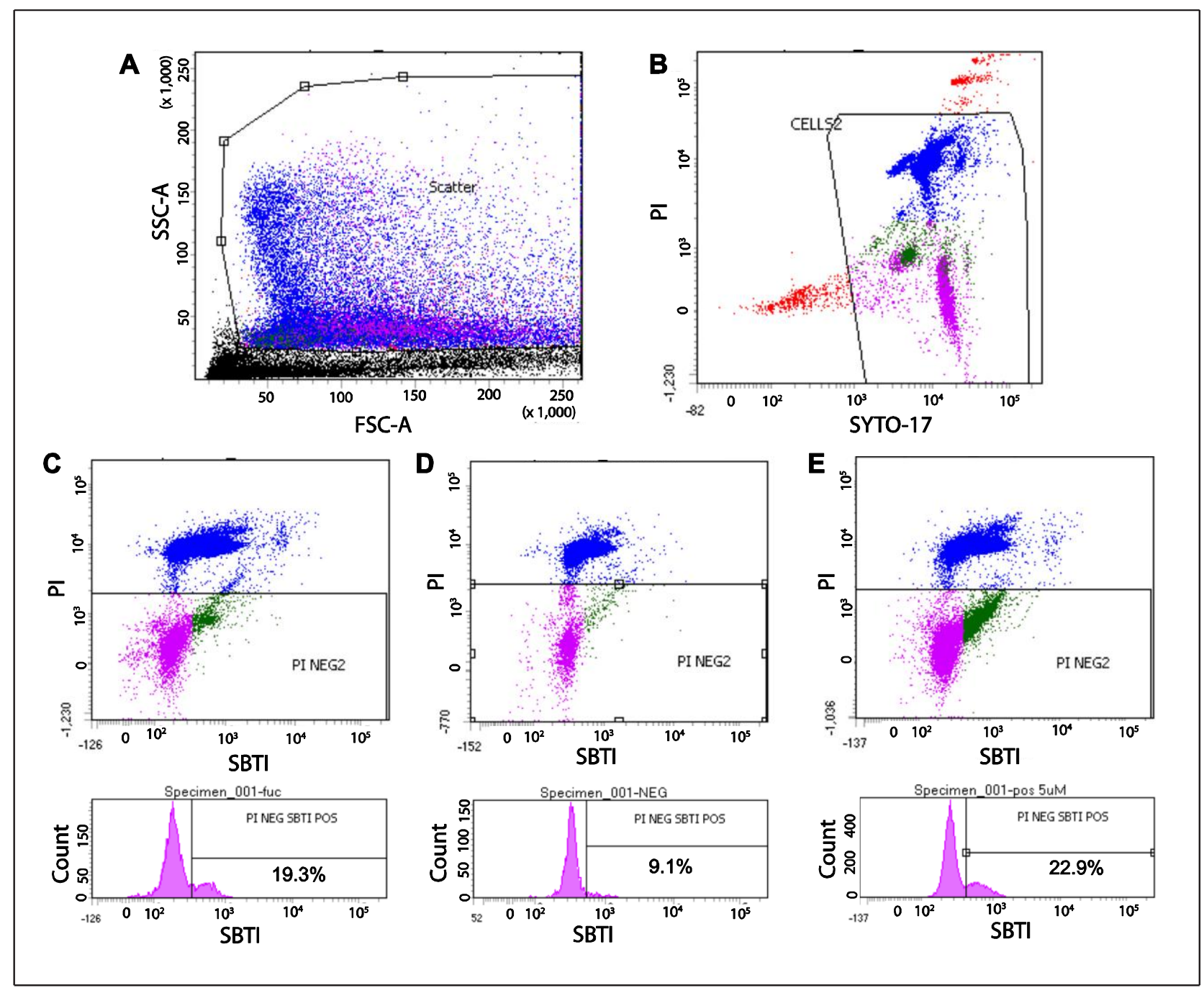

Figure 1. 


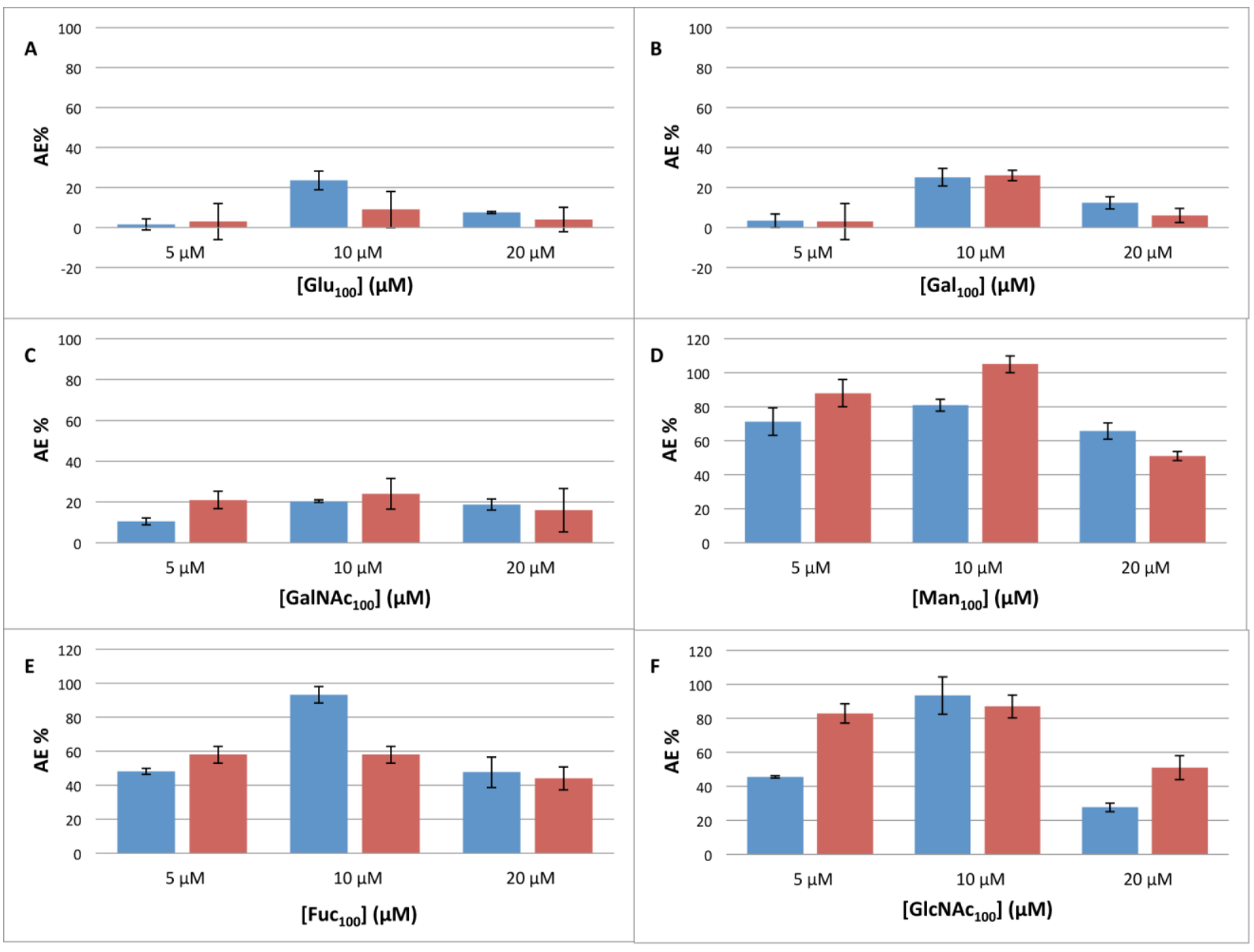

a Flow Cytometry $\quad$ Microscopy

Figure 2 


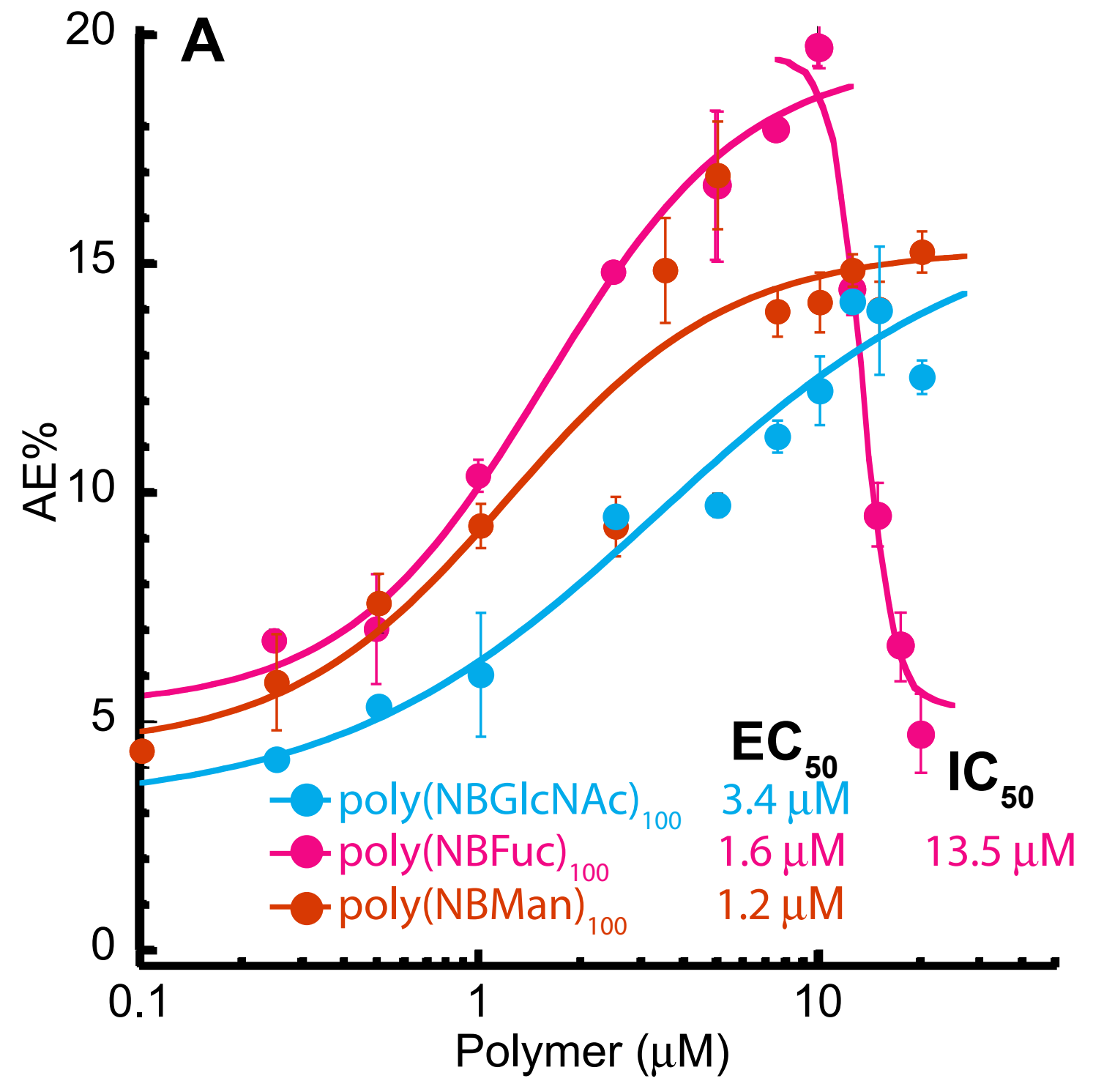

Figure 3A 

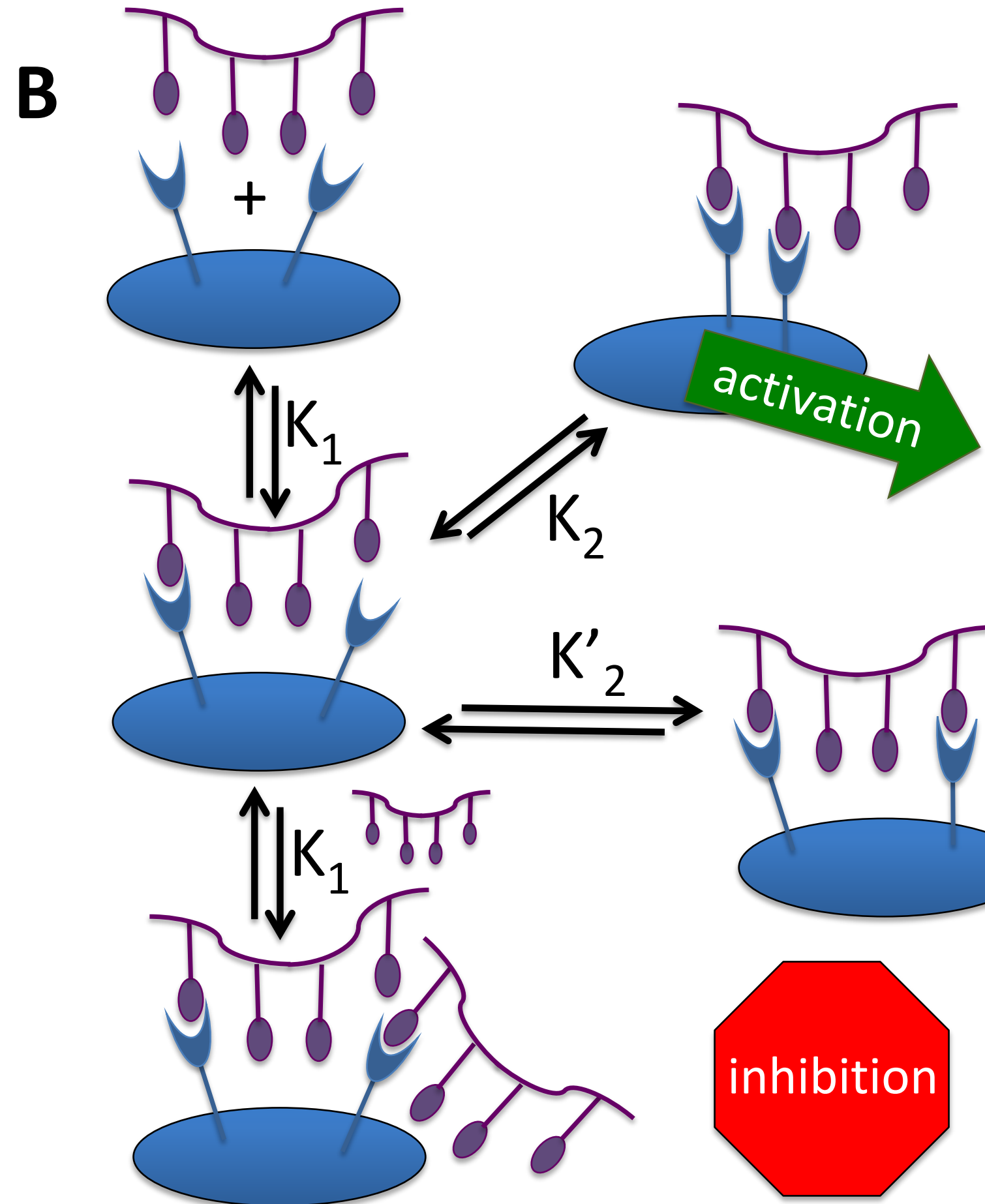

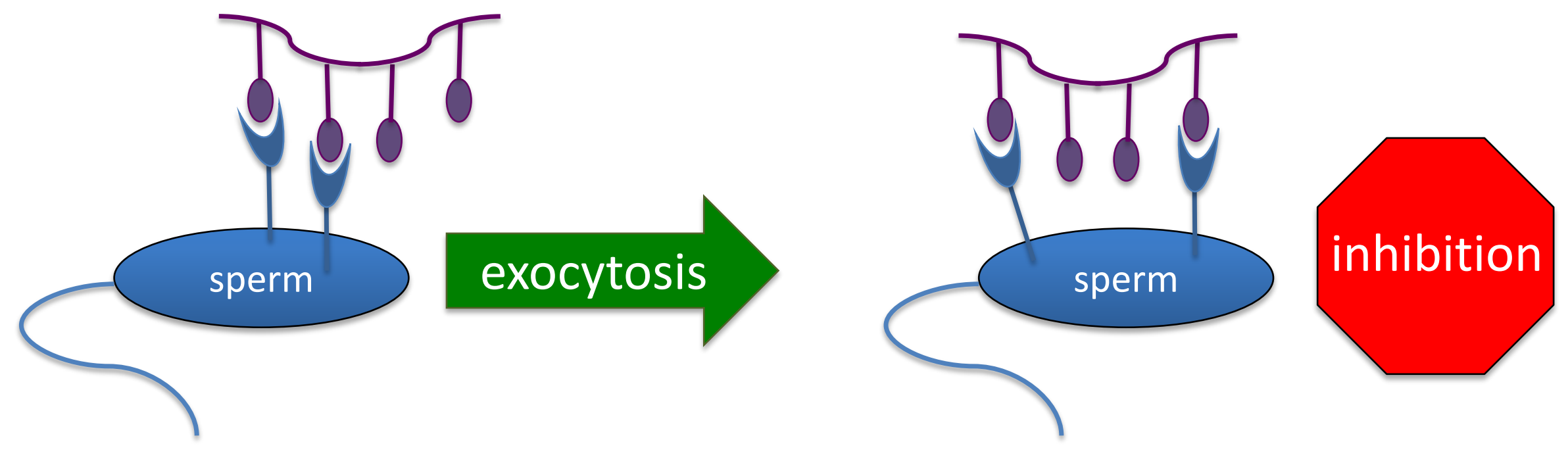

Graphical Abstract 